\title{
NOCH EINMAL DER KÖTER.
}

(Zu Beitr. 33, 402 f. und Beitr. 34, 567 f.)

Am letztgenannten orte hat Friedrich Kluge meine an H. Schroeders deutung (Beitr. 29, 554 ff.) angelehnte etymologie des ndd. nhd. köter als kauzer = 'beller' in ziemlich schroffer weise abgelehnt. Ich will daher versuchen, die an ersterem orte nur kurz angedeute etymologie hier etwas ausführlicher zu begründen.

Fr. Kluge hat darin recht, dass er in oberdeutsch kauzen, gauzen das verbalsuffix got. -atjan = ahd. -azzen (vgl. got. lauhatjan, ahd. lohazzan 'leuchten') sucht. Denn schweiz. chauze $^{n}$, chäuzen (Schweiz. idiot. 3, 595; man beachte den von Kluge vermissten umlaut sowie sein fehlen!) kann nur auf ein urgerm. *kauwatjan zurückgehen, da urgerm. *kautjan ja zu *chōzen, *chözen hätte werden müssen. ${ }^{1}$ ) - Die letztere grundform wird mit verkürzung des ahd. $\bar{o}$ vor $z$ (vgl. über diese vereinzelten fälle Wilmanns, Deutsche gramm. 12, $310 \mathrm{f}$.) in nhd. kotzen fortleben, woneben sich, besonders in der burschikosen sprache, kï̈zen findet, das wol auf md. boden entstanden ist (vgl. über solche doppelformen Behaghel, Grundriss $1^{2}, 693$ ). Ein kleiner hund, der viel bellt, heisst übrigens schweiz. chäuzerli, also $=$ hd. kauzerchen.

Das in fast allen oberd. und westmd. mundarten (nicht nur am Oberrhein, wie Fr. Kluge angibt) verbreitete kauzen, gauzen (vgl. DWb. 5, 371) findet sich im bair. auch in der form kaussen, kauschen; obersächs. liegt kauxen, gauxen vor. So werden wir auf das wurzelelement *kau- als bedeutungsträger geführt, das zweifellos onomatopoëtischen ursprungs ist. Wenn H. Hirt in der neuen auflage des Weigandschen Wb.'s I, 632 gauzen nach älterem vorgang (vgl. z. b. die citate bei Schmeller, Bair. wb. 1, 969) mit aisl. geyja, praet. gó, dän. gjo 'bellen' zusammenstellt - ae. zéađ f. 'torḩeit, leichtsinn' ist trotz anscheinender formeller übereinstimmung mit aisl. gaud f. 'das bellen' der bedeutung wegen wol fernzuhalten -, so ist dies

1) In einer mitteilung vom 19. febr. 1908 hat mich $A$ : Bachmann in Zürich hierauf bereits freundlichst hing'ewiesen. 
nur insofern richtig, als hier das im consonantischen anlaut abweichende wurzelelement ${ }^{*}$ gau - der bedeutungsträger ist. Bei einer lautnachahmenden wortbildung ist aber diese differenz (*kau- : $\left.{ }^{*} g a u-\right)$ bedeutungslos. Eine etymologische anknüpfung indes zu suchen - Hirt gibt die gleiche wie ich selbst Beitr. 33, $403-$, wird man in diesem falle doch besser unterlassen.

Den umstand, dass das auch schon aus spätmhd. zeit belegte nhd. kauzen, gauzen (kautzen Diefenbach, Gl. $70 \mathrm{~b}$ aus dem 15. jahrh., gautzen bei Alberus Dict. Q 1 a [1540], s. DWb. und Weigand a.a.o.) keine ndd. entsprechung hat, worüber sich Fr. Kluge a.a.o. wundert, erklärt H. Schroeder ganz annehmbar dadurch, dass mit der monophthongierung des au zu $\bar{o}$ auf ndd. sprachgebiet der charakteristische inhalt des wurzelelements *kau- (vgl. wau-wau) verloren gieng und es somit für den gewollten zweck unbrauchbar wurde; ein mit dem 'determinativ' $t$ erweitertes *kautan (vgl. das oben erwähnte bair. kaussen u. ähnl.) musste ja zu ndd. *kōten werden, gleichwie ein urgerman. got. *kautareis zu andd. *kōtari, *kōteri $=$ ndd. kiōter wurde.

Ich halte also meine deutung von nhd. köter als 'kauzer' immer noch für ansprechender als die ableitung von ndd. kot, engl. cot 'hütte'. Hirt hat sie übrigens im neuen Weigand s. v. loöter ebenfalls angenommen.
BERLIN N, 20. märz 1909.
S. FEIST.

\section{DAS DWB. UND DIE ZIPS.}

Weder die gebrüder Grimm, noch deren nachfolger tragen die schuld, dass in dem monumentalen werke, dem Deutschen wörterbuche, krasse fehler der nachwelt überliefert.wurden. Dieser vorwurf trifft zum grössten teile dr. K. J. Schröer, der als geborener Pressburger das ungarische bergland bereiste und sich einige tage auch in Zipsen aufhielt, worauf er in den bdn. XXV. XXVIII und XXXI der Sitzungsberichte der kais. academie der wissenschaften in Wien den Beitrag zu einem 